\title{
NUEVAS CITAS EN ELAEOCARPACEAE, ESCALLONIACEAE, ORCHIDACEAE, POLYPODIACEAE Y PTERIDACEAE PARA LA FLORA DE LA PROVINCIA DE JUJUY (ARGENTINA)
}

\author{
CLAUDIA M. MARTÍN¹ \& FREDDY BURGOS GALLARDO²
}

\begin{abstract}
Summary: Martín, C. M. \& F. Burgos Gallardo. 2015. New records in Elaeocarpaceae, Escalloniaceae, Orchidaceae, Polypodiaceae and Pteridaceae for the flora of the province of Jujuy (Argentina). Bonplandia 24(2): 81-92.
\end{abstract}

\begin{abstract}
As part of a study on the flora and vegetation from different localities in the phytogeographic province of the Yungas, we record and illustrate seven species that have not been previously reported for the Province of Jujuy (Argentina): Vallea stipularis (Elaeocarpaceae), Escallonia schreiteri (Escalloniaceae), Cyclopogon congestus, Rhinocidium fuchsii, Sacoila secundiflora (Orchidaceae), Campyloneurum angustipaleatum (Polypodiaceae) and Adiantum camptorachis (Pteridaceae).
\end{abstract}

Key words: Argentina, flora, Jujuy, Phytogeography.

\begin{abstract}
Resumen: Martín, C. M. \& F. Burgos Gallardo. 2015. Nuevas citas en Elaeocarpaceae, Escalloniaceae, Orchidaceae, Polypodiaceae y Pteridaceae para la flora de la provincia de Jujuy (Argentina). Bonplandia 24(2): 81-92.

En el marco de un estudio de flora y vegetación realizado en la Provincia Fitogeográfica de las Yungas, se citan e ilustran siete especies que no habían sido registradas para la provincia de Jujuy (Argentina): Vallea stipularis (Elaeocarpaceae), Escallonia schreiteri (Escalloniaceae), Cyclopogon congestus, Rhinocidium fuchsii, Sacoila secundiflora(Orchidaceae), Campyloneurum angustipaleatum (Polypodiaceae) y Adiantum camptorachis (Pteridaceae).
\end{abstract}

Palabras clave: Argentina, Fitogeografía, flora, Jujuy.

\section{Introducción}

En el marco de diversas campañas de colecta realizadas en la Provincia de Jujuy (Argentina), principalmente en sus zonas boscosas, se registraron siete especies que previamente no habían sido mencionadas para suflora. Elobjetivo de este trabajo es dar a conocer estos hallazgos mediante aportes morfológicos, ecológicos y extensiones del rango de distribución de las especies; por lo que resulta de interés para la fitogeografía de la provincia.

${ }^{1}$ Instituto de Bio y Geociencias del Noroeste Argentino (IBIGEO), Av. Bolivia 5150, C.P. 4400, Salta (Capital), Argentina. CONICET. claudiamartin@conicet.gov.ar

${ }^{2}$ Cátedra de Evolución, Facultad de Ciencias Agrarias, Universidad Nacional de Jujuy, Alberdi 47, C.P. 4600, San Salvador de Jujuy, Jujuy, Argentina. CIT-Jujuy CONICET. 


\section{Materiales y Métodos}

Las campañas de colecta fueron realizadas entre 2012 y 2015. Los ejemplares colectados se depositaron en el Herbario MCNS y fueron determinados mediante el uso de la bibliografía específica (Correa, 1955; Docha Neto \& Baptista, 2006; Jaramillo Azana, 1988; Johnson, 2001; Larsen et al., 2010; Novara \& Chemisquy, 2009; Stearn, 1974; Sundue, 2010; Sundue et al., 2010; Valebella \& Miguel, 2011). Se consultaron las descripciones originales $\mathrm{y}$ se estudiaron fototipos disponibles y los ejemplares depositados en los herbarios LIL y MCNS.

\section{Resultados}

Los nuevos registros para la flora de la Provincia de Jujuy, son:

\section{ELAEOCARPACEAE DC.}

Vallea stipularis L.f., Suppl. Plant. 266. 1781 (1782). Fig. 1

Arbustos o arbolitos, de aproximadamente $4 \mathrm{~m}$ de alt. Hojas simples, alternas; láminas subcordadas a cordadas de $5-7 \times 3,5-5 \mathrm{~cm}$, con ápice ligeramente redondeado, borde entero sinuoso, con 5 nervaduras principales palmadas, haz verde oscuro, lustroso, glabro, envés verde blancuzco, tomentoso en las nervaduras; pecíolo de 2-4 cm long., ensanchado en la base, pubescente. Inflorescencia en cimas dicótomas, de aproximadamente 10-12 cm long. Flores bisexuales, en pedúnculos de aproximadamente 2,5 $\mathrm{mm}$ long.; pedicelos de 1,5-3 cm long., de color rojizo en flores maduras, glabros; estípulas glabras de 0,7$1,5 \times 0,5-1,5 \mathrm{~cm}$, discoloras, verde pálido en el envés, verde lustroso en el haz. Cáliz de color rojo vinoso o rosado oscuro; con 5 sépalos libres, ovado-angostos de $6 \times 3 \mathrm{~mm}$, glabros o ligeramente pubescentes en el haz, y densamente pubescentes en los márgenes y ápice del envés. Corola rosado oscura, 4 (5) pétalos libres, de $8-10 \times 6 \mathrm{~mm}$, 3-lobados, lóbulos de 3-5 mm long., con bordes sinuosos a crenados. Androceo con numerosos estambres, aproximadamente 46; filamentos de 3-3,5 mm long., densamente pubescentes; anteras de 2,5$3 \mathrm{~mm}$ long., bitecas, con dehiscencia poricida apical. Ovario súpero, 4-locular, glabro, estilo de 4,5-5 mm long., con 5 ramas estigmáticas de aproximadamente $0,7 \mathrm{~mm}$ long. Fruto cápsula, subgloboso, con abertura 4-valvar, castaño oscuro a negruzco en la madurez. Semillas rosado-blanquecinas, de contorno ovado a redondeado, 1 por lóculo.

Nombres vulgares: "Chuillur" (Perú), "sacha capulí," "peralillo", "rosa", "cubillo", "guisho" (Ecuador), "pera caspi”, "capulí” (Bolivia). No se han registrado nombres vulgares en Argentina.

Distribución y hábitat: Se distribuye por los Andes desde Venezuela y Colombia, a través de Ecuador, Perú y Bolivia hasta Argentina (Jaramillo Anzana, 1988; Tropicos, 2015). Actualmente la especie está señalada para el NE de la provincia de Salta, Argentina (Zuloaga et al., 2015). Este registro extiende la distribución $150 \mathrm{~km}$ al sur y es el más austral para la especie. En Argentina aparece entre los 1600 y los $1900 \mathrm{~m}$ en el distrito de Bosque Montano de las Yungas (Cabrera, 1976). En Salta crece en el bosque de Alnus acuminata Kunth y Podocarpus parlatorei Pilg. (Legname, 1982), mientras que en Jujuy forma parte del bosque de Ilex argentina Lillo, Crinodendron tucumanum Lillo y P. parlatorei Pilg. Fig. 2.

Floración: Octubre-diciembre.

Material estudiado: ARGENTINA. Jujuy: Dep. Valle Grande, Abra Honda, 1900 m s.n.m., 18XI-2013, fl, Martín 250 (MCNS 12233).

Material adicional: ARGENTINA. Salta: Dep. Santa Victoria, Candelaria (ex Lapachal), 23-XI1976, fl, Giusti et al. 12077 (LIL 39176).

ESCALLONIACEAE R.Br. ex Dumort Escallonia schreiteri Sleumer, Verh. Kon. Ned. Akad. Wetensch., Afd. Natuurk., Tweede Sect. 58: 5-146. 1968. Fig. 3

Arbusto o arbolito de 2,5 m de alto, corteza castañogrisácea, ramasjóvenes levementerojizas. 

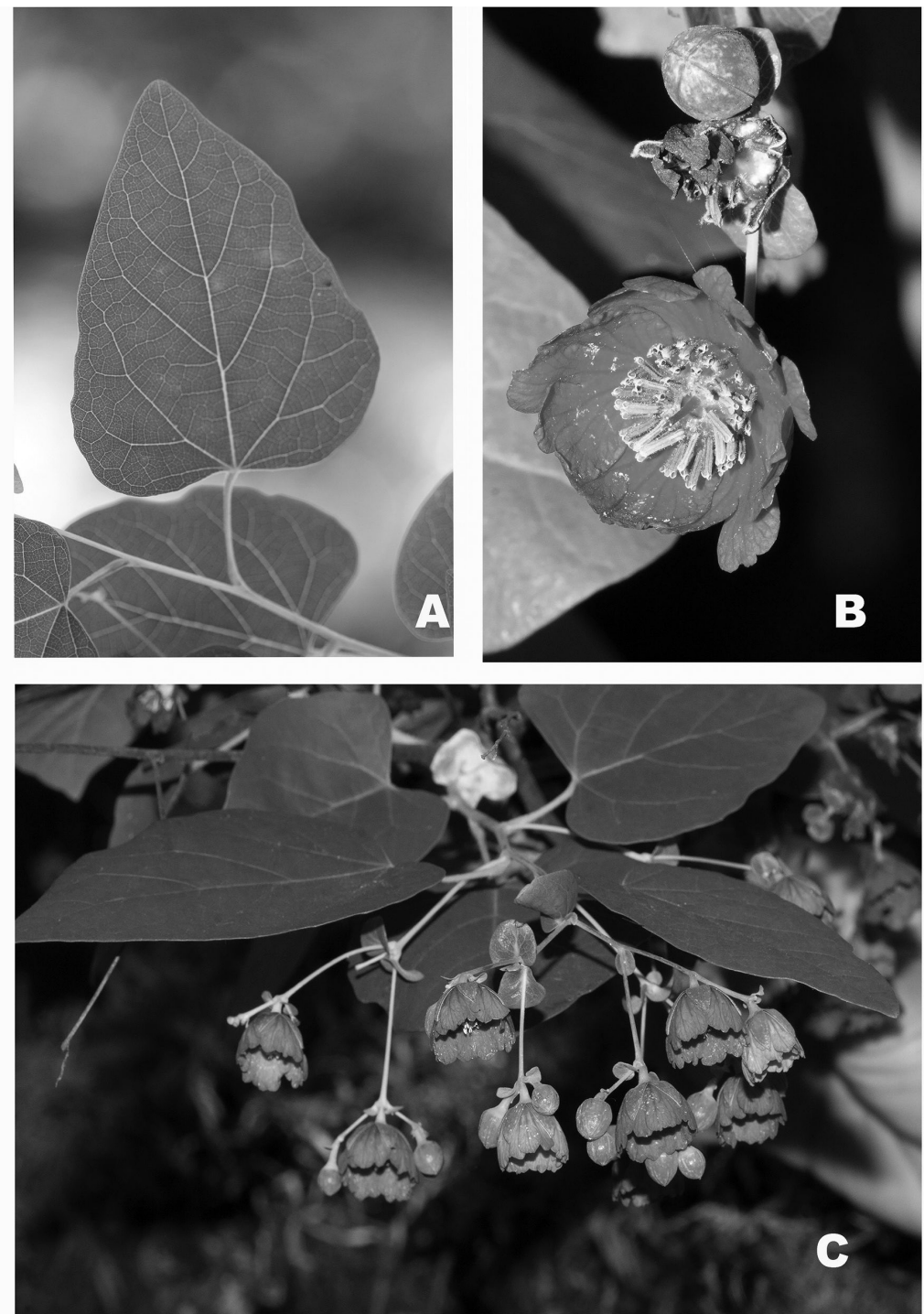

Fig. 1. Vallea stipularis. A: Hoja. B: Flor. C: Inflorescencia (Martín 250, MCNS 12233).

Hojas alternas, simples, glandulosas, lámina lanceolada de 3,5-7 × 1-2,3 cm, con ápice ligeramente acuminado, borde serrulado, base decumbente, pinnatinervada, nervio principal prominente en el envés; pecíolo de 0,6-1,2 $\mathrm{cm}$ long., levemente caniculado, laxamente pubescente. Flores bisexuales, actinomorfas y pentámeras, reunidas en panículas tirsoides terminales. Hipanto crateriforme. Cáliz con sépalos anchamente triangulares, de 1-1,5 $\times$ 1,5-2,1 mm, con tricomas en sus bordes. Corola blanca, dialipétala, pétalos lineares con ápice obtuso, de 0,75 × 0,25 $\mathrm{mm}$. Estambres alternipétalos, de 5,5-6 $\mathrm{mm}$ long., anteras oblongas, de 0,2-0,3 mm long. Ovario ínfero, nectario estilar plano, estigma peltado, bilobado, papiloso-glanduloso. Fruto cápsula, con estilo persistente.

Nombres vulgares: "Khishuara", "K'iswara", "llave", "puna lloke" (Bolivia). No se han registrado nombres vulgares en Argentina.

Distribución y hábitat: Se distribuye en Perú, Bolivia y Argentina (Gómez \& Perea, 1998; Tropicos, 2015). Hasta el momento en Argentina se la encontró en la provincia de Salta 


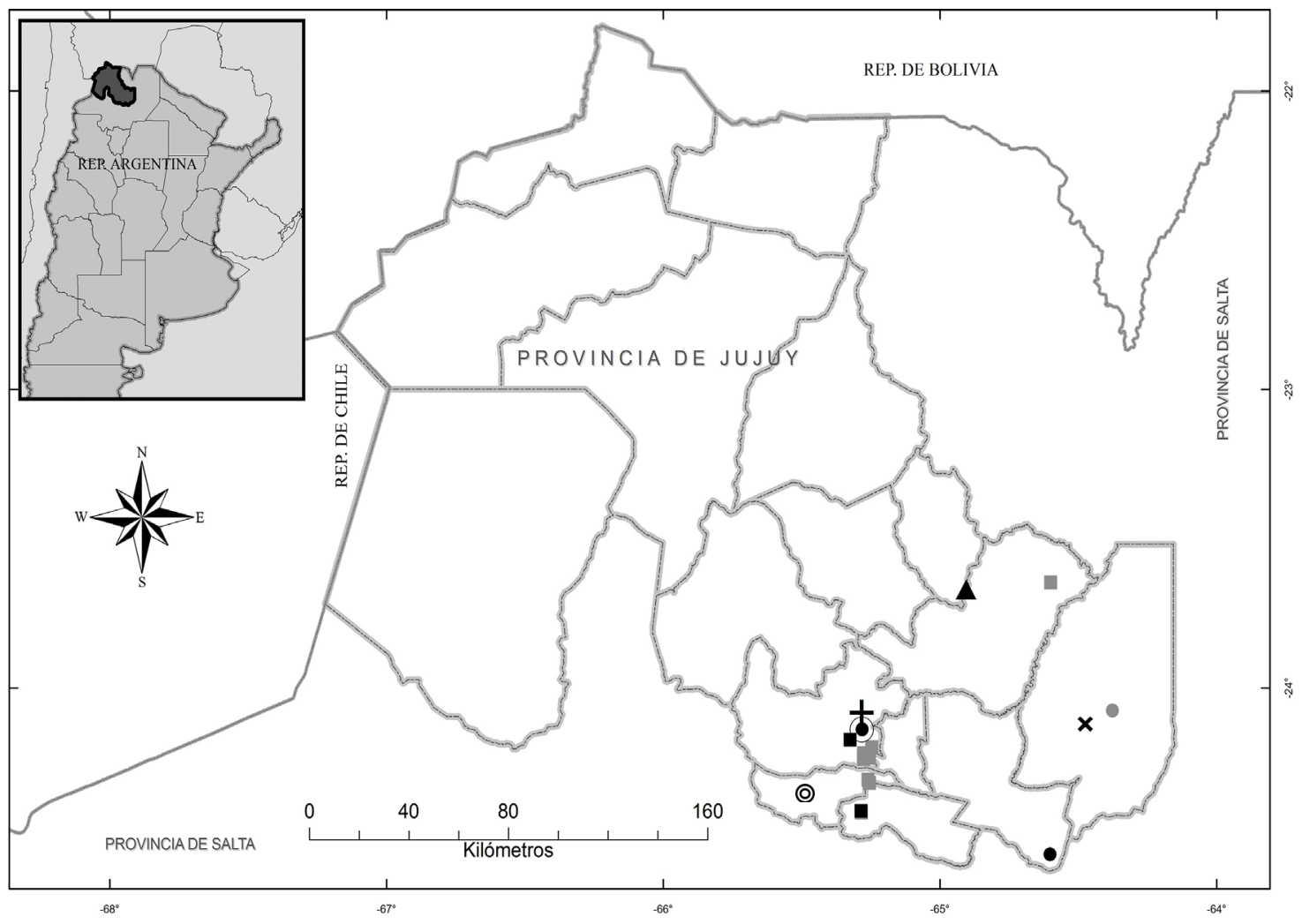

Fig. 2. Distribución geográfica de los nuevos registros para la Provincia de Jujuy: Adiantum camptorachis (círculo doble), Campyloneurum angustipaleatum (equis), Cyclopogon congestus (círculo vacío), Escallonia schreiteri (cruz), Rhinocidium fuchsii (círculo relleno), Sacoila secundiflora (cuadrado), Vallea stipularis (triángulo); en negro datos de colecta y en gris datos observados.

y Tucumán (Zuloaga et al., 2015). En Argentina ha sido hallada entre los 1200-2000 m, tanto en bosques chaqueños serranos como yungueños. Particularmente el ejemplar encontrado en Jujuy habita en el distrito de Selva Montana de las Yungas (Cabrera, 1976), en un bosque disturbado, dominado principalmente por Schinus myrtifolius (Griseb.) Cabrera y Sebastiania commersoniana (Baill.) L.B. Sm. \& Downs, específicamente entre la ruta 35 y la playa del río San José de Chijra, a la altura del puente homónimo. Fig. 2.

\section{Floración: Setiembre-diciembre.}

Obs.: Este registro eleva a 4 el número de especies de Escallonia Mutis ex L.f. presentes en Jujuy (E. hypoglauca Herzog, E. millegrana Griseb., E. schreiteri y E. tucumanensis Hosseus) (Zuloaga et al., 2015). En el campo, se podría confundir con E. tucumanensis, de la cual se diferencia por presentar el margen foliar apenas serrulado y los lóbulos calicinos anchamente triangulares.

Material estudiado: ARGENTINA. Jujuy: Dep. Dr. Manuel Belgrano, Tilquiza, cerca del puente del río Chijra, 1500 m s.n.m., 11-X-2012, fl, Martín 113 (MCNS 12874).

Material adicional: ARGENTINA. Salta: Dep. Capital, Quebrada de San Lorenzo, 28-X-1989, fl, Novara 3807 (MCNS) 08-I-1998, fr, Novara 10962 (MCNS); Dep. Guachipas, Cuesta del Lajar, 27-X-1991, fl, fr, Chaparin 23052 (MCNS); Dep. Rosario de Lerma, Quebrada del Toro, 16-I-1988, fl, fr, Novara 7506 (MCNS); 30-VI-1995, fl, Tolaba 553 (MCNS).

ORCHIDACEAE Juss.

1. Cyclopogon congestus (Vell.) Hoehne, Fl. 

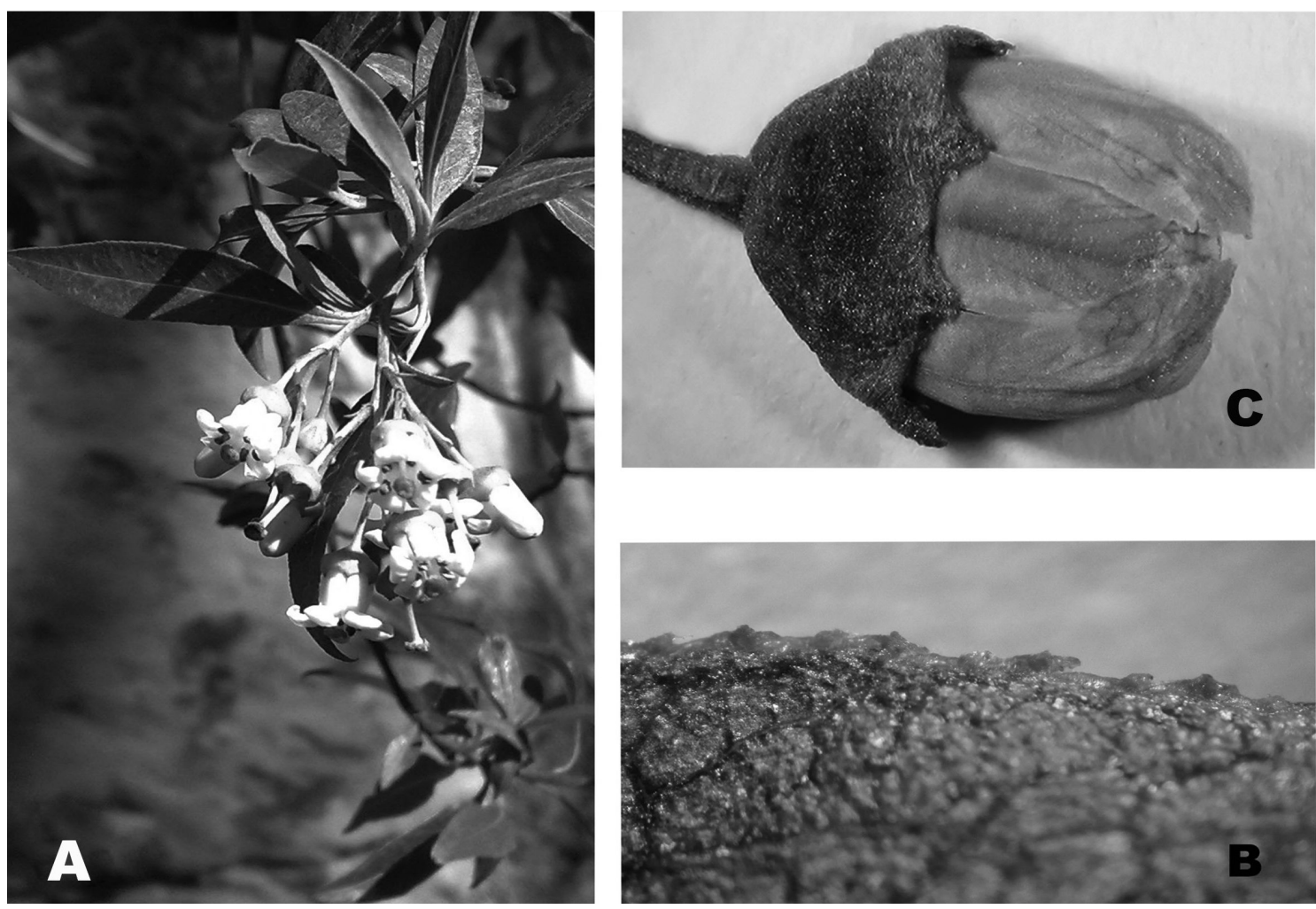

Fig. 3. Escallonia schreiteri. A: Rama florífera. B: Margen foliar. C: Detalle del cáliz en el pimpollo floral (Martín 113, MCNS 12874).

Brasilica 12: 209. 1945. Serapias congesta Vell., Fl. Flum. Icon. 9: t. 54. 1835. Spiranthes alpestris Barb. Rodr., Gen. et Spec. Orchid. Nov. 1: 184. 1877. Beadlea congesta (Vell.) Garay, Bot. Mus. Leafl. 28: 300. 1980. Fig. 4

Plantas terrestres, raro epífitas. Raíces carnosas, fasciculadas, densamente pubescentes. Hojas 6- 10, de 7-20 × 2-4 cm, oblongoelípticas, pseudopecioladas, dispuestas en una roseta basal. Inflorescencia espiciforme, 20-40 cm long., con 10-35 flores, unilaterales; vainas del escapo 7-8, acuminadas, del mismo largo o más largas que los entrenudos, glabras. Flores resupinadas, subpéndulas, perfumadas. Sépalos y pétalos verdosos a blanquecinos. Bráctea floral más larga que el ovario, acuminada, pubescente. Sépalo dorsal de 5-7 × 1,5-2,3 mm, atenuado en el ápice, pubescente; sépalos laterales de 7-7,5 $\times 1,8-2,2 \mathrm{~mm}$, con base pubescente, agudos. Pétalos de 5-6 × 1-1,2 mm, conniventes con el sépalo dorsal, ápice redondeado. Labelo blanco, de 7,5-9 × 3-4,3 mm, con 2 aurículas basales, glabro. Columna de 3,5-4 mm long., glabra. Ovario 6-7 mm long., arqueado, pubescente. Fruto cápsula elipsoide, de $7 \times 3,5 \mathrm{~mm}$.

Nombres vulgares: No se han registrado nombres vulgares en Argentina.

Distribución y hábitat: Brasil, Paraguay, Uruguay y Argentina (Johnson, 2001; Tropicos, 2015), donde está citada para las provincias de Misiones, Salta y Tucumán (Zuloaga \& al., 2015). En Jujuy aparece en el distrito de Selva Montana de las Yungas (Cabrera, 1976), en lugares húmedos y sombríos, con suelos ricos en detritos y humus en el interior del bosque de Blepharocalyx salicifolius (Kunth) O. Berg y Allophyllus edulis (A. St.-Hil., A. Juss. \& Cambess.) Hieron. ex Niederl. Fig. 2.

Floración: Junio-agosto.

Obs.: Este registro eleva a 5 el número de especies de Cyclopogon C. Presl. presentes en 


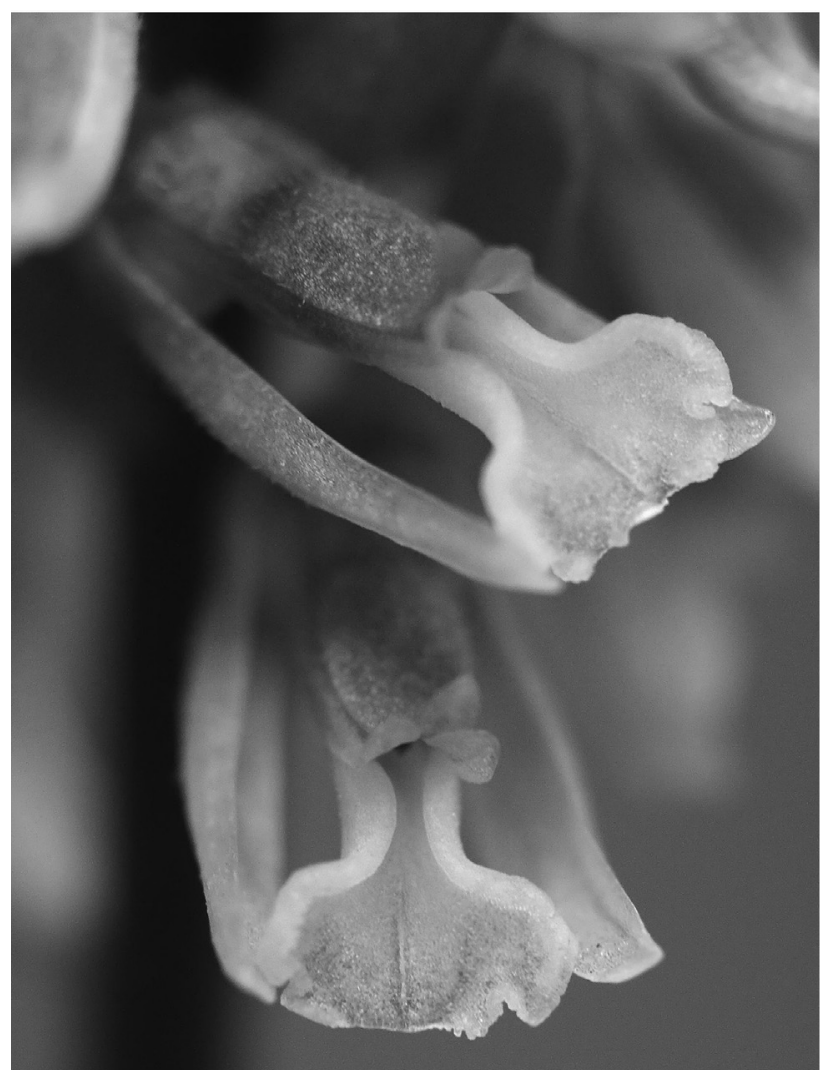

Fig. 4. Cyclopogon congestus. Flor (Martín 133, MCNS 12876).

Jujuy [C. apricus (Lindl.) Schtr., C. calophyllus Barb. Rodr., C. congestus, C. elatus (Sw.) Schltr. y C. elegans Hoehne] (Zuloaga et al., 2015). En estado vegetativo se asemeja a $C$. elatus, de la que se diferencia por presentar 6-10 hojas pseudopecioladas; vainas del escapo floral glabras, isomorfas y más largas que los entrenudos; flores vistosas, perfumadas, densamente distribuidas en la inflorescencia, con labelo blanco.

Material estudiado: ARGENTINA. Jujuy: Dep. Dr. Manuel Belgrano, San Salvador de Jujuy, Campo Verde, Finca San José de Chijra (Familia Pérez-Carillo), 1500 m s.n.m., 11-VII-2014, fl, Martín 133 (MCNS 12876).

Material adicional: ARGENTINA. Salta: Dep. La Caldera, camino a la Cornisa Salta-Jujuy, 1500 m s.n.m., 6-X-1987, Palaci 1000 (MCNS); Dep. Orán, Aguas Blancas, Finca Arrazayal, 26-VIII1986, fl, Palaci 756 (MCNS); Dep. Santa Victoria,
Parque Nacional Baritú, río Lipeo, a medio camino a Las Termas, $1200 \mathrm{~m}$ s.n.m., 3-IX-1997, fl, Johnson 776 (MCNS).

2. Rhinocidium fuchsii (Königer) Docha Neto, Orchidstud. 1: 10-12. 2006. Oncidium fuchsii Königer, W. Arcula 15: 361-363. 2005. Fig. 5

Planta epífita. Raíces blanquecinas, glabras. Rizoma reducido, raramente ramificado. Pseudobulbos de 4,5-8 cm de largo, estrechamente ovados a oblongo-ovoides, sulcados longitudinalmente, cubiertos en la base por 2-3 vainas blanquecinas, escariosas, agudas. Hojas 2-3 en el ápice del pseudobulbo, lineares, sésiles, de 10-16 × 1,5-2 cm, márgenes enteros, persistentes. Inflorescencia paniculada, de 20-35 cm long., generalmente péndula; raquis 3-5 ramoso, rama con 4-10 flores. Brácteas florales aproximadamente $2 \mathrm{~mm}$ long., subtriangulares. Flores resupinadas, de 10-14 $\mathrm{mm}$ long. Sépalos y pétalos amarillentos a 
castaño rojizos. Sépalo dorsal ovado, levemente apiculado, de 7-9 × 2,5-3,5 mm, los laterales subiguales, ovado-lanceolados, ápice agudo, $9 \times 3 \mathrm{~mm}$, ligeramente unidos en su base, con las partes libres algo divergentes. Pétalos subiguales, de 7-9 × 2,5-3 mm. Labelo trilobado, lóbulos laterales poco aparentes, ocultos casi totalmente por el disco, lóbulo medio suborbicular, de $7 \times 13 \mathrm{~mm}$, amarillo, entero, notoriamente apiculado. Columna incurvada, de 4,5 mm long. Ovario pedicelado, de 5,5-6 mm long., verde oscuro. Fruto cápsula fusiforme, $4-5 \times 1,8-2,4 \mathrm{~cm}$.

\section{Nombre vulgar: "Oberita".}

Distribución y hábitat: Se distribuye en Paraguay y Argentina (Valebella \& Miguel, 2011; Tropicos, 2015). En este último, ha sido señalada para las provincias de Salta, Tucumán y
Catamarca (Valebella \& Miguel, 2011; Zuloaga et al., 2015). Crece en borde de bosques de Yungas y sectores de transición con el Chaco Serrano (Cabrera, 1976). Particularmente en la provincia de Jujuy se la encontró como epífita de Anadenanthera colubrina (Vell.) Brenan var. cebil (Griseb.) Altschul, Tipuana tipu (Benth.) Kuntze, Jacaranda mimosifolia D. Don, Prosopis alba Griseb. y Celtis sp. Fig. 2.

\section{Floración: Noviembre-enero.}

Material estudiado: ARGENTINA. Jujuy: Dep. Dr. Manuel Belgrano, San Salvador de Jujuy, Campo Verde, Finca San José de Chijra (Familia Pérez-Carillo), 1470 m s.n.m., 9-XII-2014, fl, Martín 321 (MCNS 12873); Dep. El Carmen, Camping Municipal El Carmen, 1200 m s.n.m., 14XII-2014, fl, Martín 323 (MCNS 12877); Dep. San Pedro, San Juan de Dios, Finca Las Lauras (camino

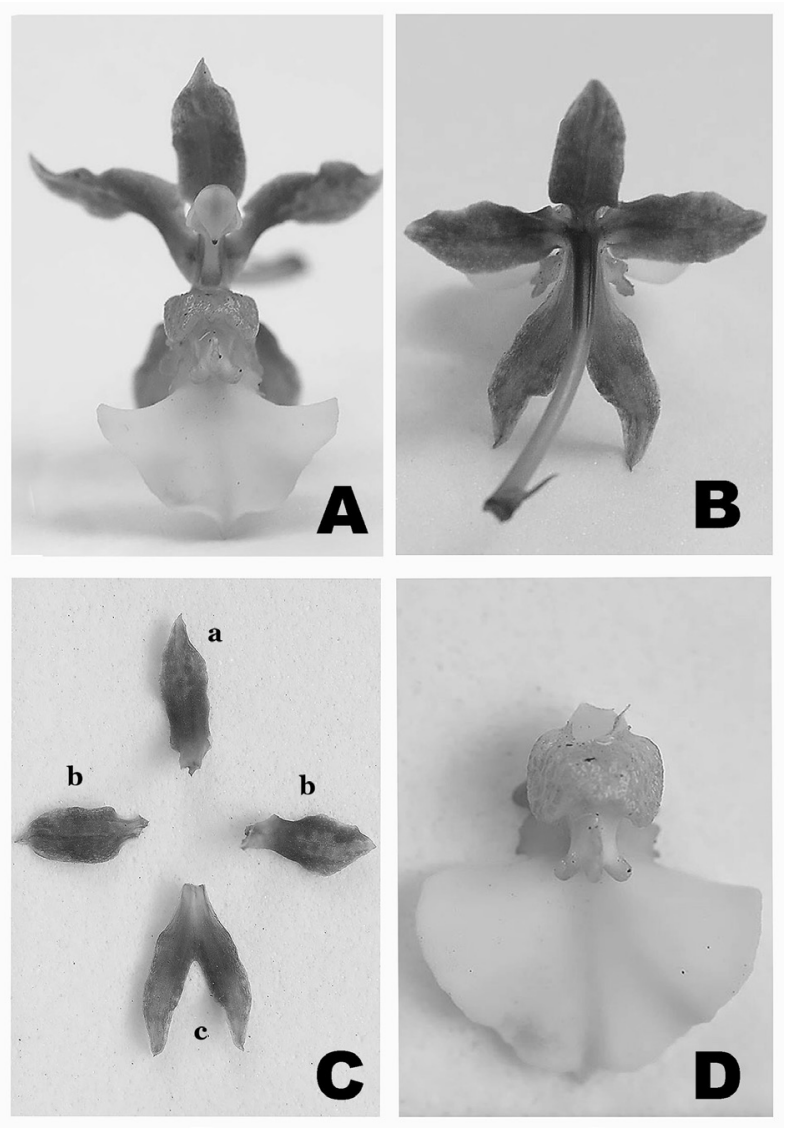

Fig. 5. Rhinocidium fuchsii. A: Flor, vista anterior. B: Flor, vista posterior. C: Flor desplegada a: sépalo dorsal, b: pétalos, c: sépalos laterales. D: Labelon (Martín 324, MCNS 12878). 
a "5 Estrellas"), 1650 m s.n.m., 11-XII-2014, fl, Martín 324 (MCNS 12878).

Material adicional: ARGENTINA. Salta: Dep. La Caldera, Abra Santa Laura, 1-XII-2010, Valebella 010-982 (CTES).

3. Sacoila secundiflora (Lillo \& Hauman) Garay, Bot. Mus. Leafl. 28: 278-425 (1982 [1981]). Stenorrhynchos secundiflorum Lillo \& Hauman, Anales Soc. Ci. Argent. 90: 134 (1921). Fig. 6

Hierbas terrestres. Raíces fasciculadas, carnosas, pubescentes. Hojas 5-7, sésiles, dispuestas en una roseta basal, presentes después de la floración, subcarnosas, de 10-30 × 5-8 cm. Inflorescencia espiciforme, de 20-45 cm long.; escapo pubescente con pelos glandulares, vainas del escapo 4-7, alternas. Brácteas florales erguidas, lanceoladas, acuminadas, más largas que el ovario. Flores resupinadas, de 18-20 mm long.. Sépalos y pétalos amarillos, muy pubescente. Sépalo dorsal libre, lanceolado, de ápice erguido; los laterales connados, lanceolados, decurrentes sobre el ovario. Pétalos lanceolados, falcados, algo decurrentes. Labelo blanco-amarillento, carnoso, lanceolado, acanalado, acuminado. Columna con un pie decurrente por el ovario. Ovario amarillo, recurvado, de 8-13,4 mm long., densamente pubescente. Fruto cápsula ovoide, de $9-16 \times 6-10 \mathrm{~mm}$.

Nombres vulgares: No se han registrado nombres vulgares en Argentina.

Distribución y hábitat: Especie endémica del noroeste argentino, señalada hasta el momento para las provincias de Salta y Tucumán (Zuloaga et al., 2015). En Jujuy, crece en el distrito de Selva Montana de las Yungas (Cabrera, 1976); particularmente en lugares abiertos con elevada insolación, es frecuente en banquinas, playa de ríos y borde de bosques. Fig. 2.

\section{Floración: Octubre-enero.}

Obs.: Este registro eleva a 3 al número de especies de Sacoila Raf. presentes en Jujuy $[S$. argentina (Griseb.) Garay, S. lanceolata (Cogn.) Schltr., S. secundiflora] (Zuloaga et al., 2015).
Material estudiado: ARGENTINA. Jujuy: Dep. Dr. Manuel Belgrano, San Salvador de Jujuy, Barrio Los Perales, 1290 m s.n.m., 24-XI-2014, fl, fr, Martín 303 (MCNS 12875); Dep. El Carmen, Camping Municipal El Carmen, 1200 m s.n.m., 12-XII-2014, fl, Martín 322 (MCNS 12879).

Material adicional: ARGENTINA. Salta: Dep. Capital, alrededores de la Universidad Nacional de Salta, 1200 m s.n.m., 28-XI-1994, fl, Fabbroni 11 (MCNS); San Lorenzo, Ruta 28, Km 4, 1400 m s.n.m. 27-XI-1999, fl, Aquino 152 (MCNS). Dep. La Caldera, Ruta 9, 1550 m s.n.m, 2-XII-1994, fl, Fabbroni 10 (MCNS); Dep. Orán, Ingenio San Martín del Tabacal, cono fluvial antiguo del río Zenta-Blanco, 7-XI-2007, fl, Zapater \& Delgado 2743 (MCNS).

POLYPODIACEAE Bercht. \& J.Presl Campyloneurum angustipaleatum (Alston) M. Mey. ex Lellinger, Amer. Fern J. 74: 56. 1984. Polypodium angustipaleatum Alston, J. Bot. 77: 346. 1939. Fig. 7

Plantas epífitas o terrestres. Rizoma rastrero, de 1,2-3 mm lat., pruinoso, escamas rizomáticas de 4,5-6,2 × 0,4-1,2 mm, patentes, castañas, linear-lanceoladas, de base redondeada a cordada, ápice largamente acuminado, clatradas, brillantes. Frondas de 12-70 cm long., muy cercanas entre sí a lo largo del rizoma; pecíolos pajizos. Láminas lineares, atenuadas en sus extremos, márgenes generalmente enteros, glabras, envés de la costa con algunas escamas linear-lanceoladas, castañas oscuras, clatradas. Soros en 1-2 filas entre la costa y el margen, sin parafisos.

Nombre vulgar: "Helecho cabello de ángel”.

Distribución y hábitat: Se distribuye desde Costa Rica, a través de Colombia, Ecuador, Perú y Bolivia hasta Argentina (León, 1993; Larsen et al. 2010; Tropicos 2015). En este último país sólo ha sido señalada para el Dep. Guachipas, provincia de Salta (Larsen et al., 2010). En Argentina crece en el distrito de Bosque Montano de las Yungas (Cabrera, 1976), en pastizales húmedos; particularmente en Jujuy vegeta entre los 2000-2500 m s.n.m, por arriba del bosque de “queñoa" (Polylepis australis Bit.). Fig. 2. 

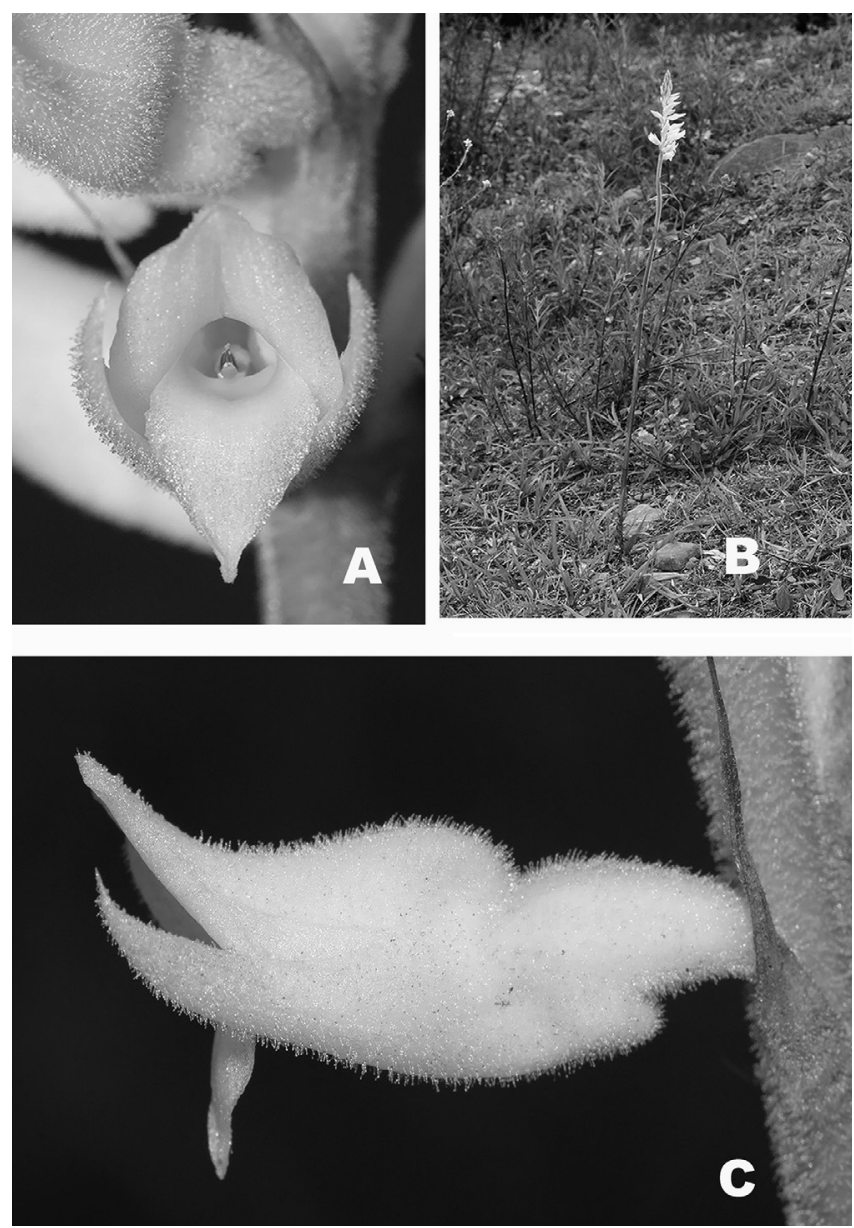

Fig. 6. Sacoila secundiflora. A: Flor, vista anterior. B: Planta. C: Flor, vista lateral (Martín 322, MCNS 12879).

Obs.: Este registro eleva a 5 el número de especies de Campyloneurum Presl. presentes en la provincia de Jujuy [C. aglaolepis (Alston) de la Sota, C. angustipaleatum, C. lorentzii (Hieron.) Ching, C. nitidum (Kaulf.) C. Presl. y C. tucumanense (Hieron.) Ching] (Zuloaga et al., 2015).

Material estudiado: ARGENTINA. Jujuy: Dep. Santa Bárbara, Cerro Bravo, 2400 m s.n.m., 24-VII-2014, Martín 140 (MCNS).

Material adicional: ARGENTINA. Salta: Dep. Guachipas, Ruta Provincial $\mathrm{N}^{\circ}$ 6, pastizal de altura, 1800 m s.n.m., 8-III-2008, Martínez 1592 (MCNS).

PTERIDACEAE E.D.M. Kirchn Adiantum camptorachis Sundue, J. Prado \& A.
R. Sm. Amer. Fern J. 100: 195-206. 2010. Fig. 8

Plantas terrestres. Rizoma largamente rastrero, de 1,5 $\mathrm{mm}$ lat., escamas rizomáticas de 1,2-2,5 × 0,3-1,2 mm, castañas, lanceoladas con ápice agudo y márgenes ciliados. Frondas de 30-65 cm long., pecíolos castaño oscuros, lustrosos, flexuosos; raquis glabro. Láminas oblongo-lanceoladas, 2-3 pinnadas, con 6-10 pares de pinnas, pínnulas de 1-1,5 × 1,5-2 cm, superficie adaxial glabra, venación flabelada, superficie abaxial con algunos pelos blancos a lo largo de las venas. Soros oblongos a reniformes, blanquecinos.

Nombre vulgar: "Culandrillo".

Distribución y hábitat: Se distribuye en 


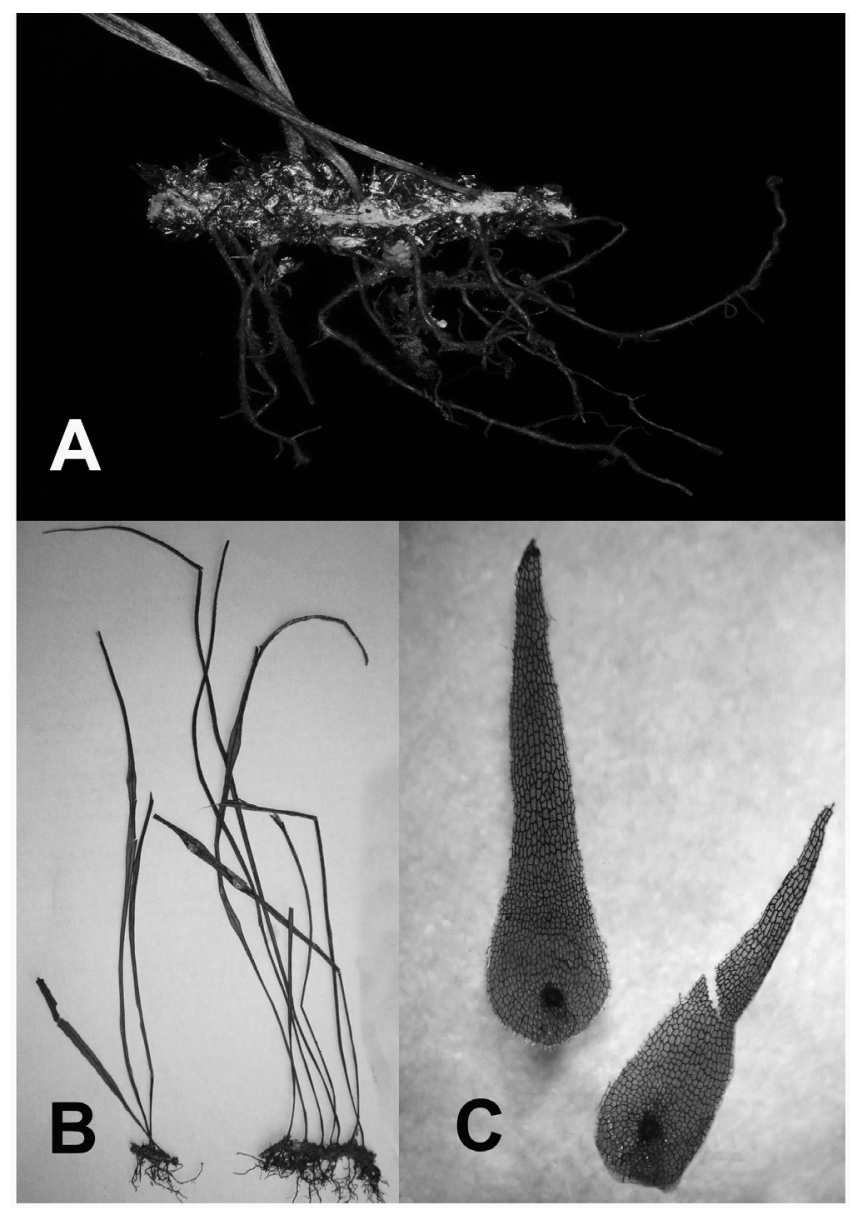

Fig. 7. Campyloneurum angustipaleatum. A: Rizoma. B: Planta. C: Escamas rizomáticas (4,5 mm long.) (Martín 140, MCNS).

Bolivia y Argentina (Sundue et al., 2010). En este último país sólo ha sido señalada para la Sierra de Achala, provincia de Córdoba (Zuloaga et al., 2015). Crece en el distrito de Bosque Montano de las Yungas (Cabrera, 1976), por encima de los $2700 \mathrm{~m}$, en pastizales húmedos.

Obs.: Este registro eleva a 9 el número de especies de Adiantum L. presentes en la provincia de Jujuy (A. camptorachis, $A$. digitatum Hook., A. lorentzii Hieron., $A$. orbignyanum Mett. ex Kuhn, A. pectinatum Kunze ex Baker, A. pseudotinctum Hieron., A. raddianum C. Presl, A. thalictroides Willd. ex Schltdl. var. hirsutum (Hook. \& Grev.) de la Sota y A. thalictroides Willd. ex Schltdl. var. thalictroides) (Zuloaga et al., 2015).
Material estudiado: ARGENTINA. Jujuy: Dep. San Antonio, Abra de Cañas, camino al Cerro Negro, 2700 m s.n.m., 18-I-2015, Martín 411 (MCNS).

Material adicional: BOLIVIA. Tarija: Prov. Arce, Municipio Padcaya, Reserva Nacional de Flora y Fauna Tariquía, bajando de la cima del Cerro Lorayoj al Abra del Lorayoj, 3200 m s.n.m., 3-VIII-2005, Serrano et al. 6148 (NY).

\section{Agradecimientos}

A la Familia Pérez-Carrillo (Finca San José de Chijra), a Ole Mustad por la hospitalidad en Las Lauras, al “Cumpa” Hugo (Q.e.p.d), Iván y José de Las Lancitas por la compañía en el campo. Al Sr. Marcelo Gerónimo (Herbario del 

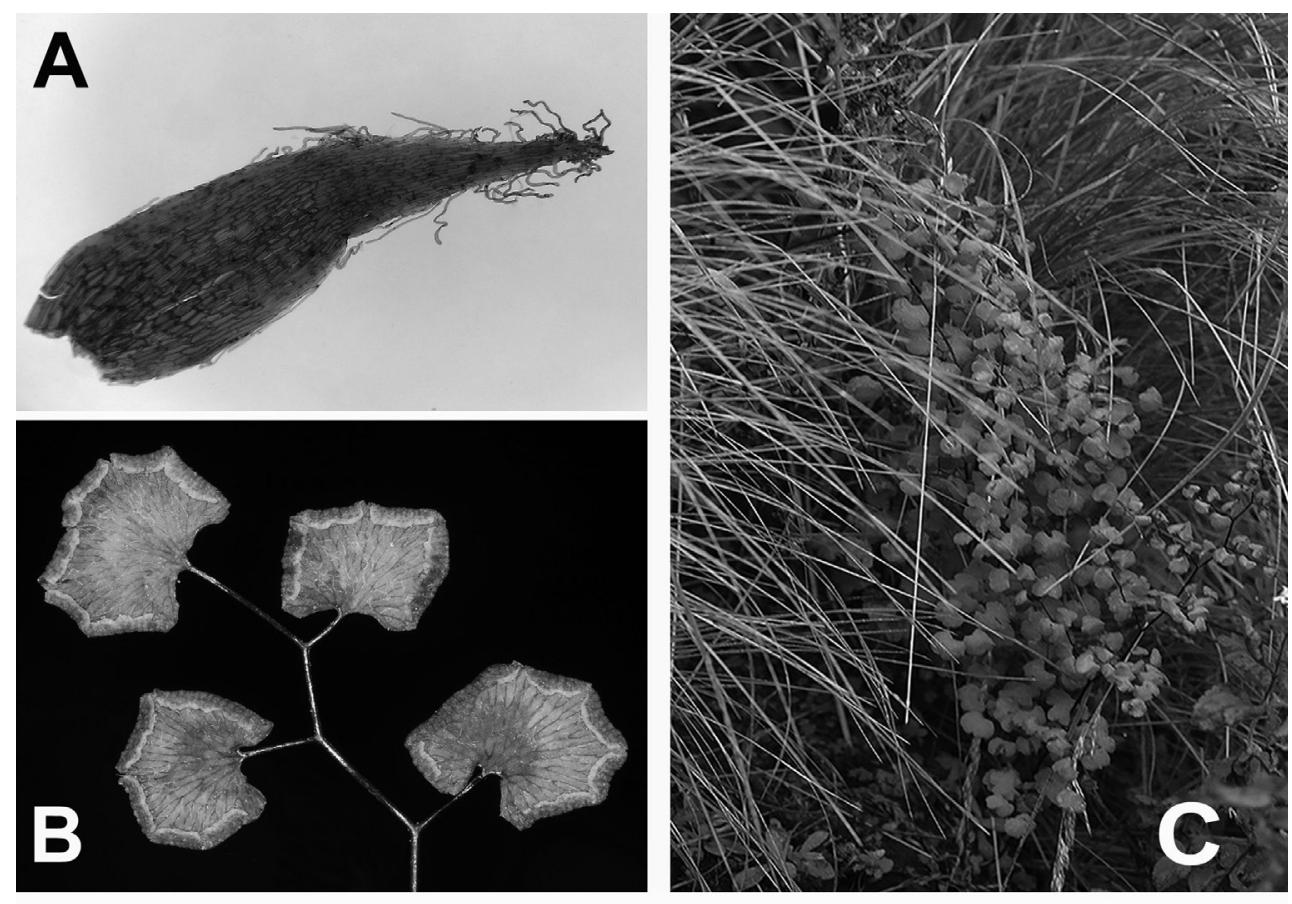

Fig. 8. Adiantum camptorachis. A: Escama rizomática (2,2 mm long.). B: Detalle de pínulas fértiles. C: Planta (Martín 411, MCNS).

Museo de Ciencias Naturales de Salta-MCNS), a la Dra. O. Martínez y a la Lic. D. Jaimez (Universidad Nacional de Salta), a Magali Méndez por la fotografía de E. schreiteri (Fig. 3: A). Al Gobierno de la Provincia de Jujuy por los permisos correspondientes y la logística (Res. N ${ }^{\circ}$ 117/2014 Secretaria de Gestión Ambiental- Dirección Provincial de Biodiversidad).

\section{Bibliografía}

CABRERA, A. L. 1976. Regiones Fitogeográficas de la República Argentina. 2da Ed. Enciclopedia de Agricultura, Jardinería y Fruticultura ACME S.A.C.I., Buenos Aires. 85 pp.

CORREA, M. N. 1955. Las Orquídeas argentinas de la tribu Polychondreae Schlechter subtribu Spiranthineae Pfitzer. Darwiniana 11: 24-88.

DOCHA NETO, A. \& E. D. H. BAPTISTA. 2006. Rhinocidium Baptista: Morfologia, ocorrência, reviscão taxónômica e nova combinacão de Orchidaceae. Orchidstudium 2: 9-16.

GÓMEZ, S. E. \& C. PEREA. 1998. Saxifragaceae Juss., en J. L. Novara (ed.), Flora del Valle de Lerma (Provincia de Salta, República Argentina). Aportes
Botánicos de Salta, Serie Flora 5: 1-11. Ed. Facultad de Ciencias Naturales, Universidad Nacional de Salta.

JARAMILLO AZANA, J. 1988. Notes on Ecuadorean Vallea (Elaeocarpaceae) with the description of a new species. Nord. J. Bot. 8: 19-23.

JOHNSON, A. E. 2001. Las Orquídeas del Parque Nacional Iguazú. L.O.L.A. 282 pp.

LARSEN, C., O. G. MARTÍNEZ \& M. M. PONCE. 2010. Nuevos registros en helechos para el noroeste de la Argentina. Darwiniana 48: 100-108.

LEGNAME, P. R. 1982. Árboles indígenas del noroeste argentino. Opera Lilloana 34: 1-226.

LEÓN, B. 1993. A taxonomic revision of the fern genus Campyloneurum. Ph.D. Thesis Aarhus University, Denmark. 119 pp.

NOVARA, J. L. \& M. A. CHEMISQUY. 2009. Orchidaceae Juss., en J. L. Novara (ed.), Flora del Valle de Lerma (Provincia de Salta, República Argentina). Aportes Botánicos de Salta, Serie Flora 9: 1-90. Ed. Facultad de Ciencias Naturales, Universidad Nacional de Salta.

STEARN, W. T. 1974. Comparative and systematic of woody Saxifragaceae. Escallonia. Bot. J. Linn. Soc. 68: $1-20$.

SUNDUE, M. 2010. Polypodiaceae, en M. H. Nee (ed.), Flora de la Región del Parque Nacional AmboróBolivia. Parte 1. Licófitas, Helechos y Gimnospermas, pp. 326-383. Santa Cruz de la Sierra: Ed. FAN. 
SUNDUE, M. A., J. PRADO \& A. L. SMITH. 2010. Adiantum camptorachis (Pteridaceae), a new species from South America with notes on the taxonomy of related species from the Southern Cone and Bolivia. Amer. Fern J. 100: 195-206.

TROPICOS 2015. Missouri Botanical Garden. Disponible en: http://www.tropicos.org Fecha de último acceso: 10 Feb 2015.

VALEBELLA, M. \& I. C. MIGUEL. 2011. Oncidium fuchsii (Orchidaceae), nueva cita para la flora de Argentina. Bonplandia 20: 41-45.

ZULOAGA, F. O., O. MORRONE \& M. J. BELGRANO (eds.). 2015. Catálogo de las Plantas Vasculares del Cono Sur (Argentina, Sur de Brasil, Chile, Paraguay y Uruguay). Disponible en: http://www2.darwin.edu.ar/ Proyectos/FloraArgentina/Generos.asp Fecha de último acceso: $10 \mathrm{Feb} 2015$.

Original recibido el 20 de abril de 2015; aceptado el 22 de septiembre de 2015. 